\title{
De la mémoire souvenir à la mémoire originaire
}

L'œuvre de Manolo Millares comme quête de l'image authentique

\section{Martine Heredia}

\section{OpenEdition}

Journals

Édition électronique

URL : http://journals.openedition.org/agedor/2449

DOI : 10.4000 /agedor.2449

ISSN : 2104-3353

Éditeur

Laboratoire LISAA

Référence électronique

Martine Heredia, « De la mémoire souvenir à la mémoire originaire », L'Âge d'or [En ligne], 3 | 2010, mis en ligne le 15 février 2010, consulté le 02 mai 2019. URL : http://journals.openedition.org/agedor/2449 ; DOI : 10.4000/agedor.2449

Ce document a été généré automatiquement le 2 mai 2019.

L'Âge d'or. Images dans le monde ibérique et ibéricoaméricain 


\title{
De la mémoire souvenir à la mémoire originaire
}

\author{
L'œuvre de Manolo Millares comme quête de l'image authentique
}

\author{
Martine Heredia
}

1 Manolo Millares (1926-1972) est surtout connu pour ses tableaux réalisés à partir de la toile à sac qu'il agglutine en leur centre, qu'il lacère, brûle, perfore ou recoud. Il la recouvre de peinture ou de goudron dans un espace qui est le lieu où se produit le combat du corps de l'artiste avec la matière. L'image produite est indéterminée et insaisissable. Elle répond au principe qui définit l'art informel et qui rejette la mimesis, cette correspondance parfaite avec la réalité. Parallèlement à ces peintures, Millares a réalisé des dessins sur un support plus souple - le papier - qu'il griffe, raye ou tache et sur lequel il dépose des images empêchant toute identification. Sur le papier, qui lui résiste tout autant, se produit le même rapport à l'espace et à la matière. Quel que soit le support, on retrouve, dans tout l'œuvre de Millares, les mêmes schémas d'organisation de l'espace, où des calligraphies énergétiques reprennent la verticalité de l'Homoncule ou l'horizontalité du Personnage tombé.

2 Or, si la volonté de nier la ressemblance, de refuser l'image identifiable, anime les peintres de l'informel - comme j'ai eu l'occasion de le démontrer dans ma thèse ${ }^{1}$ - il semblerait que la série de dessins que réalise Manolo Millares en 1971, intitulée Mussolini², soit en passe de le contredire et invite à interroger l'image chez Millares doublement. En effet, à partir du titre qui renvoie aux cadavres de Mussolini et de sa compagne Clara Petacci, exposés par les partisans à la contemplation publique, ces six dessins de petit format $(50 \times 70 \mathrm{~cm})$ nous mettent en présence d'images, au sens où on l'entend communément. Le regardeur continue de reconnaittre, plus ou moins distinctement, les traits et les formes distordus d'un corps. Donc, en nous confrontant à une variation de corps pendus par les pieds, voici encore, d'une certaine manière, une façon de reproduire, de répéter le réel, et par conséquent de démentir le principe de non-représentation. D'autre part, la référence incluse dans le titre opère un renvoi du tableau à un événement qui sollicite la mémoire historique, celle du peintre comme celle du spectateur. Alors, partant de ce réel, quel lien existe-t-il entre souvenir et fiction? Quel rapport entretient 
l'image produite par Millares avec la mémoire et l'imagination créatrice ? À quoi peut correspondre cette distorsion par rapport au principe de l'art informel? C'est ce paradoxe que je me propose d'analyser, afin d'avancer qu'en vérité, la question n'est pas de s'interroger sur la nature de l'image, mais plutôt sur sa fonction. Aussi dirons-nous, en reprenant la définition d'Henri Maldiney, que « la première fonction de l'image dans la peinture, c'est d'apparaître. Le moment esthétique n'est pas le quoi de son apparaître mais le comment de son apparition $»^{3}$. Ainsi, pour comprendre le processus créateur de Manolo Millares, il s'agira de montrer que l'image est plus qu'une chose. Pour cela, j'examinerai d'abord ce que l'image produit et, plus précisément, comment Millares met la forme en image, dans un combat avec la figure et dans un espace qui est beaucoup plus que l'espace de la toile, au sens où il englobe l'homme lui-même. Ensuite, dans une seconde étape, nous nous demanderons de quel répertoire elle surgit, afin de découvrir que l'image plastique ou picturale n'est pas une chose statique mais que, par le geste, elle est une mise en relation dynamique entre mémoire et imagination. Enfin, nous verrons que ce processus, incarné par des images, plonge dans une élémentarité qui vise à retrouver une expérience primordiale où se trouve l'image originelle.

\section{Un jeu de retournement}

3 Donc, comment Millares met-il la forme en image? Tout d'abord, je voudrais montrer qu'en passant de la toile à sac au dessin sur papier, Millares regarde le monde par une autre fenêtre. Cependant, mon hypothèse est que les motivations de la démarche créatrice sont les mêmes. Devant cette image faiblement reconnaissable que nous présente la série Mussolini, l'œil est invité à regarder moins ce qu'elle représente que ce qu'elle produit. Ici a lieu un combat avec la figure qui rejoint celui de la matière, comme dans le reste de l'œuvre de l'artiste canarien. En effet, alors que les toiles de Millares mettent en scène les matériaux dans leur épaisseur, on pourrait penser que le dessin, par l'économie de moyens utilisés, est confronté à l'impossibilité de rendre la matière. Or, ici, tout se joue autour de la figure, comme moyen de se libérer des normes de la représentation. En effet, en inversant le motif, c'est-à-dire en présentant la figure la tête en bas, l'ensemble Mussolini introduit dans l'œuvre de Millares une nouvelle tension. En vérité, il s'agit là d'une volonté d'inverser l'image au sens large, autrement dit, de bousculer le traitement traditionnel de la figure et de brouiller les codes de la perception. D'autre part, un des principes de base de l'art informel étant que la matière est à l'origine de la forme, dans ces dessins comme dans d'autres œuvres de Millares, il n'est plus question de réaliser sur la toile ou sur le papier une forme préalablement conçue, mais bien de la laisser surgir de la matière. Au-delà des signes iconiques, l'inversion de la figure est donc révélatrice du travail de Millares, de sa volonté de faire du corps, la matrice qui donnera naissance à la forme, de son désir de permettre à la forme de s'inventer, de mettre en image l'informulé. Le rapprochement entre la figure des dessins Mussolini et celle des tableaux Anthropofaune, Homme de Neandertal ou Homoncule, rend manifeste cette intention. En d'autres termes, le retournement de la figure permet son détournement, au sens où il donne la possibilité de retourner vers l'œuvre en soi, de conduire le regard vers le trait associé à l'encre, en même temps que vers le geste. Ainsi, la violence résultant de la distorsion faite à la figure est une base qui stimule l'acte de créer de Millares, comme l'est parallèlement la surface de la feuille de la série Mussolini pour le geste qui, dans un même temps, s'affirme et se nie. En effet, de l'encre de chine, à 
coups de traits hachurés, de ratures, de signes qui occultent ou effacent, de taches sur la surface blanche du papier comme s'il s'agissait de brouillon ou d'esquisse, jaillissent des créatures à mi-chemin entre l'humain et l'animal, s'avançant par deux ou par groupes compacts, parcourues de mouvements rapides. Comme dans sa peinture, le mouvement est nerveux, empreint d'automatisme. Millares laisse aller sa main pour libérer le geste ; car l'image est bien un faire, elle n'a pas son fondement en elle-même, elle est une construction après coup.

4 Si le trait renvoie au geste, il renvoie au corps, c'est-à-dire à une force, à une action visible. Paul Klee, déjà, l'incluait dans les «matériaux » nécessaires à la réalisation d'une œuvre, précisant que cette force, propre à chaque artiste, doit se faire reconnaître dans la matière, dans laquelle elle prend corps, devient forme et réalitét ${ }^{4}$. La force qui mobilise le corps, nous la trouvons dans l'œuvre de Millares dont les toiles à sac, tordues à pleines mains, témoignent de l'effort physique qui entraîne tout le corps. Les dessins, quant à eux, laissent apparaître la trace qui rend manifeste la présence de l'acte. La force transparaît alors dans l'action visible de la rature: Manolo Millares nous donne à voir l'effort qu'il fait pour effacer et gommer le trait, en même temps que l'œuvre en train de se faire. Cette force implique de tenir compte de la spontanéité du geste qui met le corps en tension. Ce qui importe, c'est le rapport de l'artiste à l'espace, car l'image se réalise dans une agitation de surface, mais aussi dans l'espace d'un acte humain. Le fait est que l'espace informel a changé de sens; il n'est plus le milieu dans lequel se disposent les choses, mais, comme dirait Merleau-Ponty, «le moyen par lequel la disposition des choses, la puissance universelle de leurs connexions, devient possible $»^{5}$. En vérité, l'espace n'est pas seulement une chose devant Millares; il est comme un milieu qui l'enveloppe et le pénètre et dans lequel il est au monde à travers la motricité de son corps. Parce qu'il peut se déplacer avec plus d'aisance que le sculpteur qui, lui, est souvent ralenti par la masse de la pierre ou du métal qu'il manipule, le geste du peintre est plus libre. Cependant, cette liberté n'est pas seulement un signe de l'homme; elle est l'acte même de l'homme. Par cette liberté, l'artiste est souvent démiurge ; néanmoins il ne jouit pas entièrement de ce pouvoir, puisque, dans l'art informel, il lui faut compter avec la nature de la texture qu'il manipule et qui répond à la tension de l'artiste dans un rapport de réciprocité. Autrement dit, par l'extrême proximité qui s'établit entre les matériaux et l'acte pictural, l'espace de la toile ou de tout autre support - en l'occurrence, le papier- est l'espace du vécu, celui où l'homme se manifeste; c'est l'espace du vide où se réalise le passage entre l'invisible - qui correspond à la sphère personnelle de l'artiste - et le visible - qui est de l'ordre du tableau lui-même - , là où sont amenés à la surface les courants invisibles intérieurs.

\section{L'image comme médiatrice}

De quel répertoire surgit alors l'image chez Manolo Millares? Dans le cas présent, à travers les corps pendus par les pieds, comme l'animal aux crochets de boucherie, l'image de la série Mussolini nous met en présence de "l'abominable réalité ", celle qui renvoie à des images appartenant à un patrimoine commun : celui de notre mémoire, la mémoire historique, celui aussi d'un musée imaginaire de l'horreur, comme s'y est risqué en son temps Jean Fautrier avec les Otages (1945). Cette série, exécutée pendant la Seconde Guerre Mondiale, tentait de suggérer l'horreur uniquement par le travail de l'artiste, visible sur les couches de peinture grattées, griffées, traversées dans tous les sens. 
L'exécution, insolite à l'époque, défiait la tradition, faisant voler en éclats la figuration ; aux abominations de la Deuxième Guerre mondiale, qui avait dépassé la Première, et à la barbarie de l'inhumanité, Fautrier répondait par la barbarie de l'exécution. Autrement dit, comme celles de Fautrier, les images de cette série de Millares replongent dans l'archaïsme - le nôtre et celui de l'humanité - ; elles nous placent devant toute la barbarie dont est capable ou victime notre monde. N'oublions pas que l'art informel - qui est celui de Millares, mais aussi de Tàpies et de Saura ${ }^{6}$ - est né d'un contexte douloureux, laissé par les séquelles de la Seconde Guerre mondiale, d'un temps où, après les abominations dont a fait preuve la civilisation, les peintres viennent à douter de l'humanité et prennent conscience que les problèmes que soulève l'art ne peuvent plus se résoudre dans une iconographie $r$ : "l'harmonie n'est plus de notre temps », écrit Millares dans une lettre de El Pas? . Savoir que Manolo Millares réalise le cycle Mussolini en 1971 et non pas dans les années de l'après-guerre, inciterait à rejoindre Valeriano Bozal selon lequel «le passé n'a pas disparu, il reste présent, il est présent ${ }^{8}$. Cependant, j'ajouterai que, si l'œuvre de Manolo Millares pointe son rapport au réel vécu, à l'histoire qui est bien celle de ce contexte et de cette œuvre-là, la peinture de Millares est, comme le dit Tió Bellido, " autre chose qu'un témoignage documentaire " ${ }^{9}$; autrement dit, ce qui s'y passe est au-delà du caractère anecdotique, au-delà de la caricature. L'image ici n'est pas chose passive emmagasinée dans la mémoire mais elle est un "entre-deux", fonctionnant comme médiatrice. En effet, par «l'actualisation du souvenir en sensations naissantes et en mouvements commencés $»^{10}$, comme l'énonce Bergson dans L'énergie spir ituelle, le souvenir est riche en germes qui peuvent donner naissance à des créations - et non à de simples reproductions- en étant activées par des images dans l'acte corporel. L'image est alors le lien entre le passé et le présent, entre mémoire et imagination créatrice. Ce processus est incarné par les images qui, elles-mêmes, sont intégrées dans un dynamisme; et c'est de là que naîtra la création. En d'autres termes, les images de Millares ne replongent pas seulement dans un passé vécu; en ayant recours au geste irréfléchi et rapide, l'acte de peindre correspond à un désir de ne conserver que le fond élémentaire du rythme et par là, Millares plonge dans ce répertoire d'images qui appartiennent à l'imaginaire, région commune à tous les hommes, en l'occurrence, à l'espèce humaine. Ces images deviennent des archétypes, c'est-à-dire des schèmes de signification générale, qui résonnent en chacun de nous et font sens pour tout le monde. La question qui se pose maintenant est de savoir comment est ranimée cette zone de l'imaginaire, commune à tous, et quel travail elle déclenche.

\section{Faire éclater les couches de surface}

6 Le point d'émergence de l'œuvre d'art a toujours quelque chose de mystérieux ; Paul Klee, déjà, avait souligné que celui-ci semble être une projection du «fond originel supradimensionnel $\aleph^{11}$ qui, même à l'état latent, est source de fécondité ; aussi peut-on lire dans sa Philosophie de la création:

L'œuvre est au premier chef genèse et son histoire peut se représenter brièvement comme une étincelle mystérieuse jaillie d'on ne sait où qui enflamme l'esprit, actionne la main et, se transmettant comme mouvement à la matière, devient

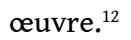

7 En retrouvant le mouvement préalable, en laissant se développer une œuvre très simple, primitive, Paul Klee montre que l'artiste sent s'éveiller sa disposition créatrice qui le 
mobilise, pour mobiliser à son tour. Les peintres informels ont été sensibles à cette conception de la création et cherchent de même à retrouver un état de nature garant d'authenticité. Ainsi, le processus de création permet de retourner à cette expérience primordiale à travers les archétypes symboliques, ces sortes de symboles fondamentaux servant de matrices à des séries de représentation. Jung, dont l'influence auprès des artistes de l'informel est reconnue, en a souligné l'importance, en montrant que tous les inconscients individuels s'enracinent dans un inconscient collectif. Les archétypes ont, en effet, une puissance esthétique certaine, d'abord par le fait qu'ils ébranlent en profondeur la sensibilité, ensuite par leur caractère de "contagiosité»: celui qui est ému profondément par l'archétype est apte à ébranler, de même, autrui. En d'autres termes, l'image présentée permet de faire remonter à la surface les couches qui sont profondément enfouies au fond du regardeur. Il semble qu'ainsi se profile une conception de l'art que partagent bien des peintres de l'après-guerre, comme l'atteste un texte que Jean Dubuffet écrit en 1951 :

Ce que nous attendons d'une œuvre d'art, c'est que son auteur y ait découvert, y ait inventé des moyens de faire éclater ses couches de surfaces, et de livrer passage aux voies de ses couches sous-jacentes - et par là même aussi du même coup des nôtres dès que cette œuvre est devant nos yeux. ${ }^{13}$

De ce face-à-face avec les mécanismes profonds, naîtra une révélation qui jettera une lumière sur l'être de l'observateur et sur le monde, et qui aura pour conséquence de lui faire voir les choses d'une autre façon. Cette conception s'inscrit dans la perspective de l'esthétique de l'art informel et de la définition établie par Michel Tapié, critique et théoricien français qui, le premier, emploie le terme d'informel en 1948. Telle est donc la raison d'être de l'art, que ne manque pas de partager Manolo Millares. Par les procédés utilisés et le recours au geste irréfléchi, l'artiste canarien tente de rejoindre une conception primitiviste de l'art où le sentir l'emporte sur le savoir, où ce qui prime est un stade de vie vécue comme nature. C'est ainsi qu'il compte trouver l'image originelle qui va nourrir les images "personnelles » à partir d'un fond archaïque. Millares explore ce dernier dès 1949, au début de sa trajectoire, devant les pictogrammes des Indiens guanche $s$, premiers habitants des Canaries. L'artiste canarien ne se départira jamais de cette élémentarité originaire, comme l'a déjà souligné Valeriano Bozal ${ }^{14}$. Vingt ans après les « $P$ ictographies ", Millares rejoint sa quête du primitif, depuis les Homoncules jusqu'aux « Anth ropofaunes » et « Néanderthaliens». Si les premiers renvoient aux alchimistes qui pensaient transmuter le plomb en or et produire la pierre des philosophes ou l'hermaphrodite, être réunissant à lui seul le masculin et le féminin, et si les seconds sont des créatures de l'artiste démiurge, à mi-chemin entre l'humain et l'animal, tous évoquent non seulement le primitif mais l'espèce. Ils manifestent que le germe de l'esprit serait à découvrir dans la matière et le germe de la matière dans l'esprit. Cette primitivité dans laquelle plongent les œuvres de Millares est à même de renvoyer à une primitivité plus universelle. Ainsi, l'acte de peindre apparaît comme activité magique, capable de métamorphoser la masse informe en image; Antonio Saura partage la même conception: «Conduire l'image du support vers sa métamorphose pour atteindre l'archétype $»^{15}$, écrit-il dans un texte de Mé moire du temps. Car c'est dans la «source» que se trouve l'énergie nécessaire, indispensable à l'évolution intérieure, qui permettra au peintre de renaître et déclenchera en lui la mise en route de nouveaux processus créateurs en atteignant un état de fusion entre l'esprit et la matière. Pour Millares, peintre de l'Homoncule, l'œuvre est l'espace de transformation, à partir d'éléments de la conscience qui paraissent 
chaotiques et qui, dans une sorte de voyage intérieur, font irruption sur le support, grâce à la création plastique, qui est commencement d'un ordre de la massa confusa ${ }^{16}$.

Ranimant cette zone de l'imaginaire commune à tous les hommes, le principe qui meut donc la démarche de Millares est celui de la transformation qui ramène l'homme à luimême, en même temps qu'elle le fait se sentir partie prenante d'un monde qui nous englobe tous. Plus que d'un retour aux origines, il est question pour Millares de se construire un monde à lui, à travers sa propre archéologie, en passant du chaos au cosmos qu'il enfantera. Dans le geste, dans l'acte de peindre, il déforme et reconstruit l'univers, "transformant la matière en énergie cosmogonique " ${ }^{17}$, écrira en écho Antonio Saura. Il est donc question de plonger dans un répertoire commun, car il est plus facile, semble-t-il, de rejoindre à travers l'expérience picturale, le primitif et le primordial, d'entrer en contact avec ce que l'artiste a de plus vrai en lui. L'archétype chez Millares, comme chez d'autres peintres informels, n'est pas une chose mais une fonction: il provoque un certain travail. Il crée un déséquilibre pour enclencher un effort et donc une dynamique de pensée chez le récepteur qui tentera de percevoir le même chemin que l'artiste. Par conséquent, l'image est bien plus qu'une chose, elle est un intermédiaire entre la matière, qui se laisse voir, et l'esprit. On est tenté alors de rejoindre la conception de Bergson selon laquelle les images doivent servir non pas à exprimer mais à suggérer, ce qui permet de faire vivre l'expérience (que l'artiste veut exprimer) pour elle-même, comme, par exemple, la musique parvient à le faire ${ }^{18}$. La suggestion est alors réellement métaphysique : elle est dynamique et créatrice. L'image de Millares est active et forme une étincelle pouvant déclencher une dynamique chez le regardeur qui doit sortir du confort de ses habitudes de perception. Il s'agit pour l'artiste de communiquer une expérience en créant un véritable mouvement spirituel.

Pour conclure, les dessins Mussolini ne démentent donc pas le principe de nonreprésentation qui anime l'art informel et le reste de la production de Millares. Ainsi, de même que l'art informel est absence de forme mais pas pour autant sa négation, de même il y a bien refus de représentation du réel mais présence d'un autre type de représentation qui puise dans la mémoire collective pour alimenter les «images personnelles » afin de créer des images nouvelles. Éclairée par l'ensemble des œuvres de l'artiste, la série Mussolini permet de mettre en évidence la cohérence de l'ensemble de sa création artistique. Manolo Millares ne cherche pas à véhiculer le poids du souvenir, mais à créer une nouvelle expression qui s'invente à mesure qu'elle s'élabore. Dessin et peinture semblent être approche hésitante de l'être, partant du même refus de l'imitation, du même projet de donner forme à l'informe. Cette trajectoire révèle par là même que l'image n'est pas quelque chose de fixe, mais bien une dynamique entre souvenir et fiction, entre mémoire et imagination, seule condition pour que la création telle que la conçoit Millares, c'est-à-dire en quête d'authenticité et d'innocence, soit possible. 


\section{NOTES}

1. HEREDIA, Martine, L'Art Informel en Espagne : d'une praxis à la formulation d'une expérience du mond $e$, Thèse de Doctorat sous la direction de Nancy Berthier, Paris, Université Paris-Est, LISAA, EA 4120, 2009.

2.

3. MALDINEY, Henri, Regard Parole Espace, Lausanne, édit. L'Âge de l'Homme, 1999, p. 106.

4. KLEE, Paul, Théorie de l'art moderne, [1912], Paris, Gonthier, 1964, p. 54.

5. MERLEAU-PONTY, Maurice, Phénoménologie de la perception, [1945], Paris, Gallimard, édit. 2005, p. 290.

6. Manolo Millares, Antoni Tàpies et Antonio Saura sont les trois artistes qui forment le corpus de mon travail de Thèse de doctorat.

7. MILLARES, Manolo, «El Paso, 3. Carta de noviembre de 1958 », in El Paso, 1957-1960, Antológica, Caja de Pamplona, 1998, p. 29 et sui V.

8. BOZAL, Valeriano, El tiempo del estupor, Madrid, Siruela, 2004, p. 16 : “el pasado no ha desaparec ido, se mantiene como presente, es presente".

9. TIÓ BELLIDO, Ramón, « L’Histoire, Millares », in Manolo Millares (1926-1972), Toulouse, Musée des Augustins, 1982, p. 26.

10. BERGSON, Henri, L'énergie spirituelle, [1919], Paris, PUF, 1990, p. 155.

11. KLEE, Paul, Théorie de l'art moderne, [1912], op.cit. p. 59.

12. Ibid.

13. DUBUFFET, Jean, « Honneur aux valeurs sauvages », in L'homme du commun à l'ouvrage, [1973], Paris, Gallimard, collection Folio Essais, 1991, p. 95.

14. BOZAL, Valeriano, Pintura y escultura española del siglo XX (1939-1990), 1939-1990, Madrid, Espa sa Calpe, Summa Artis, 1993, p. 395.

15. SAURA, Antonio, « Superposiciones », in Note Book (memoria del tiempo), Valencia, Artes Gráficas Soler, 1992, p. 85.

16. Voir la 3 partie "La création comme expérience" de ma thèse de doctorat, HEREDIA, Martine, L'Art Informel en Espagne : d'une praxis à la formulation d'une expérience du monde, op.cit.

17. SAURA, Antonio, « Damas ", in Note Book (memoria del tiempo), op. cit, p. 55.

18. BERGSON, Henri, Essai sur les données immédiates de la conscience, Paris, PUF, 1970, p. 11-12.

\section{RÉSUMÉS}

Alors que, généralement, les tableaux de Millares renvoient une image insaisissable, les dessins sur papier de la série Mussolini nous mettent en présence d'images, au sens où on l'entend communément. Orienté par le titre, l'observateur continue de reconnaître, plus ou moins, les traits distordus d'un corps. Il s'agit ici de montrer que, quel que soit le support, les motivations de la démarche créatrice de Millares sont les mêmes : l'œil est invité à regarder moins ce que l'image représente que ce qu'elle produit. Ici, se joue un combat avec la figure qui rejoint celui de la matière, partant du même projet de donner forme à l'informe. L'image de Millares n'est pas 
chose passive, emmagasinée dans la mémoire, mais bien un intermédiaire entre souvenir et imagination créatrice. En puisant dans la mémoire collective, que le geste permet d'actualiser, Millares cherche à retourner à une expérience primordiale, où se trouve l'image originelle qui va nourrir sa création.

Mientras que, por lo general, los cuadros de Millares presentan una imagen indefinible, los dibuj os sobre papel de la serie Mussolini nos ponen en presencia de imágenes, en el sentido común de 1 a palabra. Orientado por el título, el observador sigue reconociendo, más o menos, las formas ret orcidas de un cuerpo. Me propongo mostrar que, cualquiera que sea el soporte, las motivaciones del proceso creador de Millares son las mismas: se invita al ojo a mirar menos lo que la imagen re presenta que lo que produce. Aquí, ocurre un combate con la figura que remite al de la materia, y que parte del mismo proyecto de dar forma a lo informe. La imagen de Millares no es cosa pasiva, almacenada en la memoria, sino un intermediario entre memoria e imaginación creadora. Sumié ndose en la memoria colectiva, que el gesto permite actualizar, Millares trata de volver a una exp eriencia primordial, en la cual se encuentra la imagen original que va a alimentar su creación.

\section{INDEX}

Palabras claves : Millares, pintura siglo XX, imagen, informal, gesto, memoria

Mots-clés: Millares, peinture XXe siècle, image, informel, geste, mémoire

\section{AUTEUR}

\section{MARTINE HEREDIA}

Université Paris-Est LISAA, EA 4120 